\title{
Entwicklung einer Methodik für die Ausarbeitung von Leitlinien für optimale medizinische Praxis
}

Empfehlung Rec(2001)13 des Ministerkomitees des Europarates an die Mitgliedstaaten über die Entwicklung einer Methodik für die Ausarbeitung von Leitlinien für optimale medizinische Praxis*

Das Ministerkomitee, gemäss Artikel 15.b der Satzung des Europarates,

in der Erwägung, dass es das Ziel des Europarates ist, eine engere Verbindung zwischen seinen Mitgliedern herzustellen, und dass dieses Ziel unter anderem durch gemeinsames Handeln im Gesundheitsbereich erreicht werden kann;

in Anbetracht der Bestimmungen der Konvention zum Schutze der Menschenrechte und Grundfreiheiten und der Europäischen Sozialcharta;

unter Hinweis darauf, dass Artikel 3 des Übereinkommens über Menschenrechte und Biomedizin von den Vertragspartnern verlangt, «gleichen Zugang zu einer Gesundheitsversorgung von angemessener Qualität» zu schaffen, dass Artikel 4 des gleichen Übereinkommens verlangt, dass jede Intervention im Gesundheitsbereich einschliesslich der Forschung, nach den einschlägigen Rechtsvorschriften, Berufspflichten und Verhaltensregeln erfolgen muss und dass Artikel 10 das Recht jedes einzelnen auf Auskunft in Bezug auf alle seine Gesundheit betreffenden Informationen betont;

eingedenk der Empfehlungen des Ministerkomitees an die Mitgliedstaaten Nr. R (97) 5 über den Schutz der medizinischen Daten, Nr. R (97) 17 über die Entwicklung und Schaffung von Systemen zur Qualitätsverbesserung in der Gesundheitsversorgung, Nr. R (99) 21 über die Kriterien für das Führen von Wartelisten und Wartefristen in der Gesundheitsversorgung sowie Nr. R (2000) 5 über die Entwicklung von Strukturen für eine Beteiligung des Bürgers bzw. Patienten an Entscheidungsprozessen, die die Gesundheitsversorgung betreffen;

in Anerkennung, dass sich die Gesundheitspolitik und die Gesundheitssysteme auf die besten verfügbaren Erkenntnisse (die sogenannte «Evidenz») stützen sollten;

in Anerkennung, dass die in Leitlinien entkomitee, am 10. Oktober 2001 an der 768. Sitzung der Ministerdelegierten. nalen Entscheidungen über die Aufstellung von
Bedarfsprioritäten im Gesundheitsbereich unter Berücksichtigung der ethischen, sozialen und finanziellen Fragen, der strukturellen Unterschiede zwischen den Gesundheitssystemen und den Schwankungen der epidemiologischen und gesundheitlichen Daten - unterstützen können, jedoch nicht zur reinen Kostendämpfung oder zu Rationierungszwecken verwendet werden sollten;

in Anerkennung des Rechts der Patienten und Bürger auf schlüssige und leicht zugängliche Informationen über ihre Gesundheit und über ihre Gesundheitsversorgung in einer für sie verständlichen Form und Sprache;

in der Erwägung, dass die Grundsätze einer optimalen medizinischen Praxis auf die Primär-, Sekundär- und Tertiärversorgung und auf alle Gesundheitsberufe sowie auf Gesundheitsförderung, Prävention, Diagnostik, Behandlung, Rehabilitation und andere Aspekte der Gesundheitsversorgung gleichermassen anwendbar sind;

in Anerkennung, dass Leitlinien für optimale medizinische Praxis in verschiedenen Ländern auf unterschiedliche Weise in einem komplexen durch das Gesundheitssystem sowie durch ethische, ökonomische, soziale, legale und andere Faktoren geprägten Umfeld entwickelt werden;

in Erwägung, dass die Methodik der Entwicklung und Implementierung von Leitlinien nationale Grenzen überschreitet, und dass die Evaluation und Interpretation der Belege (Evidenz) ausreichende Ressourcen und Sachkenntnisse erfordern und gemeinsam durchgeführt werden sollten;

in Anerkennung der Notwendigkeit, eine Harmonisierung der nationalen und internationalen Vorschriften zur Qualitätsforschung und zur angewandten klinischen Forschung zu fördern;

in Anerkennung, dass Leitlinien nur ein Mittel unter anderen sind, um die Qualität und die Angemessenheit der Gesundheitsversorgung zu verbessern, und daher weder ein fundiertes klinisches Urteil ersetzen können noch an die Stelle der beruflichen Verantwortung der Leistungserbringer oder der Präferenzen der Patienten treten können; 
in Erwägung, dass der Hauptzweck von Leitlinien die Unterstützung und Förderung einer guten klinischen Praxis im besten Interesse der Patienten ist und dass diese deshalb als gesundheitspolitisches Instrument angesehen werden sollten, dessen Interpretation und Rechtsstellung von der eigenen Situation des jeweiligen Landes abhängt,

empfiehlt den Regierungen der Mitgliedstaaten:

i. einen kohärenten und umfassenden Rahmen für die nationalen politischen Konzepte und Massnahmen zu schaffen, der:

- sicherstellt, dass die nationalen Methoden zur Erarbeitung und Evaluation von Leitlinien für optimale medizinische Praxis den international akzeptierten und gegenwärtig modernsten Vorgehensweisen entsprechen,

- sicherstellt, dass die Entscheidungsträger, die Angehörigen der Gesundheitsberufe, die Bürger und die Patienten die Vorteile anerkennen, die sich aus der Verwendung der besten verfügbaren Erkenntnisse (Evidenz) als Informationsgrundlage für medizinische Entscheidungen ergeben,

- die Erstellung, Verwendung und rechtzeitige Fortschreibung national und lokal bedeutsamer, evidenzbasierter Leitlinien für die klinische Praxis und für medizinische Behandlungsstrategien zu wichtigen Problemen der Gesundheitsversorgung unterstützt,

- sicherstellt, dass Leitlinien unter Berücksichtigung der ihnen innewohnenden rechtlichen Aspekte erarbeitet und implementiert werden,

- sicherstellt, dass Leitlinien sachgerecht implementiert werden und ihre Wirkungen auf die medizinische Vorgehensweise und deren Resultate sowie auf die rechtlichen Folgen für Patienten und Erbringer medizinischer Leistungen überwacht werden,

- den Zugang zu Leitlinien und deren Anwendung sowie $\mathrm{zu}$ Informationen über ihren Zweck, ihren Rechtsstatus und ihre Rechtsfolgen ebenso erleichtert wie die Verfügbarkeit von Publikationen zur Gesundheitsversorgung und elektronisch verfügbaren Informationen für Bürger, Patienten und Fachkräfte in verständlicher Sprache und in benutzerfreundlichem Format;

ii. die internationale Vernetzung von Organisationen, Forschungseinrichtungen, Clearingstellen und anderen Institutionen, die evi- denzbasierte, medizinische Informationen erarbeiten, zu fördern;

iii. die aktive und gezielte Verbreitung dieser Empfehlungen und des Erläuternden Memorandums $\mathrm{zu}$ unterstützen und dabei die Personen und Organisationen besonders zu berücksichtigen, die an Entscheidungen im Gesundheitswesen beteiligt sind.

\section{Anhang zur Empfehlung $\operatorname{Rec}(2001) 13$}

\section{Leitlinien als Unterstützung der Gesund- heitsversorgung}

Vorrangiges Ziel von Leitlinien ist die Unterstützung und Förderung guter medizinischer Praxis.

Leitlinien werden im komplexen Umfeld eines Gesundheitssystems mit seinen ethischen, ökonomischen, gesetzlichen und anderen Aspekten erarbeitet und verwendet; diese Aspekte sollten in jedem Land berücksichtigt werden.

\section{Themenauswahl}

Die Themenauswahl sollte sich danach richten, dass die zu erarbeitenden Leitlinien die Entscheidungsfindung in wichtigen Fragen der Gesundheitsversorgung unterstützen und fördern.

Die Priorisierung von Leitlinienthemen kann vorgenommen werden aufgrund der Epidemiologie von Gesundheitsproblemen, von Ungleichheiten im Gesundheitsbereich, von Schwankungen bei der Bereitstellung und der Qualität der Versorgung, aufgrund der Entwicklung neuer Technologien oder anderer Faktoren, die einen Bedarf an qualitativ hochwertigen und aktuellen Informationen entstehen lassen.

Das Vorhandensein von bereits verfügbaren evidenzbasierten Leitlinien sollte bei der Themenpriorisierung für zu erarbeitende Leitlinien berücksichtigt werden.

\section{Erarbeitung von Leitlinien}

Leitlinien sollten systematisch, unabhängig und transparent und unter Verwendung geeigneter Qualitätskriterien von Arbeitsgruppen entwickelt werden, die sich aus Fachleuten verschiedener Berufssparten zusammensetzen.

Bevor eine Leitlinie zur Implementierung freigegeben wird, ist die Beteiligung der Leitlinienanwender - durch umfassende Begutachtung und / oder durch Testung der Pilotversion - notwendig.

Werden Leitlinien aus anderen Ländern oder Regionen übernommen, müssen sie überarbeitet und neu redigiert oder auf ihre Anwendbarkeit im neuen Umfeld geprüft werden. 


\section{Verbreitung von Leitlinien}

Die Finanzierung der Verbreitung, Implementierung, Evaluation und Fortschreibung sollte zum Zeitpunkt der Entscheidung für eine Leitlinienentwicklung sorgfältig berücksichtigt werden. Es können verschiedene Finanzierungsformen existieren. Die Finanzierungsquelle muss transparent sein.

Leitlinien sollten verschiedene Zielgruppen ansprechen (Angehörige der Gesundheitsberufe, Patienten und Entscheidungsträger), und sie sollten in zielgruppengerechten Formaten verfügbar sein.

Die Verbreitung von Leitlinien sollte geplant, aktiv und nachhaltig sein und einen leichten Zugang zu Leitlinien sicherstellen.

Leitlinien-Clearingstellen und Programme zur Erarbeitung von Leitlinien erleichtern den Zugang zu verschiedenen Leitlinien für gleiche Fragestellungen und können die Qualität von Leitlinien verbessern.

\section{Implementierung von Leitlinien}

Um die Leitlinienimplementierung möglichst wirkungsvoll zu gestalten, sind systematisches Qualitätsmanagement der Gesundheitsversorgung und die Festlegung von Verantwortlichkeiten essentiell.

Zur Sicherstellung einer maximalen Leitlinienwirkung sollten verschiedene Verbreitungs- und Implementierungsstrategien miteinander kombiniert werden.

Bei der Leitlinienanwendung müssen sowohl auf nationaler als auf lokaler Ebene positive und negative Anreize (und zwar berufliche, organisatorische, finanzielle und regelsetzende) zusammen mit anderen hemmenden und begünstigenden Faktoren bedacht werden (massgeschneiderte Implementierung).
Bei der Leitlinienimplementierung hat das Interesse des individuellen Patienten im Mittelpunkt zu stehen, ausserdem sind die Berufspflichten der Leistungserbringer und die Patientenrechte zu respektieren.

Leitlinien müssen ein wesentlicher Bestandteil sowohl von Grundausbildung und klinischer Ausbildung der Angehörigen der Gesundheitsberufe als auch der kontinuierlichen Fort- und Weiterbildung von multiprofessionellen Teams werden.

\section{Evaluation von Leitlinien und ihrer Auswirkungen}

Die Festlegung, welche Leitlinien implementiert werden sollen, sollte unter Verwendung von Instrumenten zur Qualitätsbewertung bestehender Leitlinien erfolgen.

Eine wohl durchdachte Überwachung von Leitlinienwirkungen, insbesondere der Auswirkung von Leitlinien auf Gesundheitsversorgung und Krankheitsverlauf, ist von wesentlicher Bedeutung.

Leitlinien können wesentliche Indikatoren nennen, die zur Evaluation der Ergebnisse der Leitlinienimplementierung verwandt werden können.

Ein auf internationaler Ebene koordiniertes Forschungsnetzwerk sollte die Methodik der Leitlinienevaluation und der Überprüfung ihrer Auswirkungen, einschliesslich des Einflusses auf den Lernprozess und die medizinischen Kenntnisse des Fachpersonals, untersuchen.

\section{Fortschreibung}

Im Rahmen der Leitlinienerarbeitung sind klare Strategien und Verantwortlichkeiten für die Leitlinienfortschreibung festzulegen. 